\title{
Counter-propagating plasma jet collision and shock formation on a compact current driver
}

\author{
J. C. Valenzuela, G. W. Collins IV, T. Zick, J. Narkis, I. Krasheninnikov and F. N. Beg
}

Center for Energy Research, University of California-San Diego, La Jolla, CA 92037, USA

In this paper we report on the ability of a compact current driver yielding $250 \mathrm{kA}$ in 150 ns to produce counter-propagating plasma flows. The flows were produced by two vertically-opposed conical wire arrays separated by $1 \mathrm{~cm}$, each comprised of 8 wires. With this array configuration, we were able to produce two supersonic plasma jets with velocities on the order of $100-200 \mathrm{~km} / \mathrm{s}$ that propagate towards each other and collide. Aluminum wires were tested first; we observed a shock wave forming at the collision region that remained stationary for an extended period of time ( $50 \mathrm{~ns}$ ) using optical probing diagnostics and Extreme Ultraviolet imaging. After this period, a bow shock is formed that propagates at $20 \mathrm{~km} / \mathrm{s}$ towards the cathode of the array, likely due to small differences in the density and/or speed of the jets. The inter-jet ion mean free path was estimated to be larger than the shock scale length for aluminum, indicating that the shock is not mediated by collisions, but possibly by a magnetic field, whose potential sources are also discussed. Radiative cooling and density contrast between the jets were found to be important in the shock wave dynamics. We studied the importance of these effects by colliding jets of two different materials, using aluminum in one and copper in the other. In this configuration, the bow shock was observed to collapse into a thin shell and then to fragment, forming clumpy features. Simultaneously, the tip of the bow shock is seen to narrow as the bow shock moves at a similar speed observed in the Al-Al case. We discuss the similarity criteria for scaling astrophysical objects to the laboratory, finding that the dimensionless numbers are promising.

Key words: laboratory astrophysics, conical wire array, plasma jets, radiative cooling.

\section{Introduction}

Collimated outflows, or jets, are produced by astrophysical objects such as planetary nebulae[1], active galactic nuclei[2], and young stellar objects[3] on various scales, possessing a diverse set of physical parameters and features. Irrespective of their differences, much remains to be learned about these outflows - specifically their formation and the effect of an ambient medium on their propagation. Several studies have been done using numerical simulations[4,5], and recently some work has been done with scaled experiments in the laboratory to improve our understanding of the outflow dynamics.

Depending on the governing physics, some similarity criteria can be established between astrophysical and laboratory plasmas. In order to apply the ideal hydrodynamic equations, the systems in question must have the following: a localization parameter $\delta<<1$, Reynolds number $R_{e}>1$, and Peclet number $\mathrm{P}_{\mathrm{e}}>>1$. If these conditions are met, the two systems will evolve similarly when they have the same Euler number $\left(\mathrm{E}_{\mathrm{u}}\right)$, provided similar initial conditions $[6,7]$. If the physics is governed by ideal magnetohydrodynamics (MHD), which requires that the magnetic Reynolds number be $R_{\mathrm{eM}}>1$, we must also have the $\beta$ parameter (ratio of thermal to magnetic pressure) be the same for the systems to be scalable. Observations indicate that radiative cooling significantly affects the behavior of astrophysical jets[8,9], this is quantify by the cooling parameter, $\chi$, defined as the ratio of the radiative cooling time to the hydrodynamic timescale, which is less than 1 for radiatively cooled systems. Hence, if we want to include radiation in scaled experiments, an additional dimensionless number must be equal in both systems. Ryutov et al.[10] gives an expression for this dimensionless parameter when radiative cooling has a simple powerlaw type function with respect to pressure and density, although it is usually a complex form of the 
arguments. If the radiative losses in an astrophysical system can be approximated by a power-law function, it can easily be reproduced in the laboratory system.

Laboratory experiments have been performed in recent years using high-intensity lasers[11-13] and pulsed power facilities[14-16] to produce supersonic jets with scaling criteria to jets emanating from young stellar objects (YSO). Particularly in pulsed power, work has been done on the interaction of supersonic, radiative cooling jets with a cross wind[17]. The deflection of the jet observed in their work was consistent with simulations and developed models on astrophysical jet deflection. Some work has also been done on the interaction of plasma jets with a quasi-stationary background medium in order to replicate astrophysical jets propagating through the interstellar medium. Features resembling Herbig-Haro objects were identified[18,19].

In this paper, we show preliminary results on the interaction of counter-propagating plasma jets using a small current driver. Shock formation is studied and the relevance to astrophysical YSO is discussed. The configuration can be easily modified to vary parameters such as radiative cooling by using high atomic number materials or jet density by varying the array diameter. This configuration is also suitable for collisionless shock studies, where the underlying physics is not yet well understood[20,21]. While jet-related work has been done on experiments using high-intensity laser and large pulsed power facilities, these experimental resources are beyond the scope of most university laboratories, emphasizing the critical importance of studying such phenomena in small-scale experiments for the development of the field.

The remainder of this paper is organized as follows: in section 2, we have outlined the experimental setup of the colliding plasma jets and diagnostics. In section 3 we present experimental data on the shock formation and evolution. In section 4 we discuss the relevance of this work for astrophysical phenomena. In section 5 we summarize our findings and address future work.

\section{Experimental setup}

This work was performed on GenASIS, a compact, low-inductance current driver capable of producing $250 \mathrm{kA}$ in about $150 \mathrm{~ns}$ on a short circuit load at a nominal voltage of $75 \mathrm{kV}$. The details of the driver can be found in Ref. [22]. Fig. 1 shows a schematic of the load configuration used to produce two counter-propagating plasma jets. Due to the dimensions of the array, which has a larger inductance than the short circuit configuration, the driver current decreased to a peak value of 190-200 kA. The setup consisted of two opposing conical wire arrays driven by the same current pulse. The physics of conical wire arrays has been broadly studied[15,16]. Ohmic heating from the current passing through the wires quickly melts down and vaporizes them, forming a low-density coronal plasma around a high density core. At this point, the current flows mainly in the coronal plasma. The Lorentz force produced by interaction between the selfgenerated global magnetic field and the current density drives the coronal plasma towards the array axis, perpendicular to the axis of each of the wires. As the plasma stagnates on axis, it produces a standing conical shock that drives a plasma jet away from the cone's apex. The plasma jet is accelerated mainly by two mechanisms: the aforementioned standing shock that diverts the plasma flows coming from the wires in the axial direction and the plasma pressure gradient along the standing shock. In our setup, two plasma jets are produced, propagating in opposite directions corresponding to the orientation of the two arrays. The middle electrode seen in Fig. 1, which provides the arrays' aperture, is held by two plastic insulators against the top electrode in order to provide full current through the top array. These electrodes were attached to each other using only two posts in order to have a larger field of view of the collision region. We also used $5 \mathrm{~mm}$ diameter lead apertures on each of these electrodes in order to assure that the plasma jets 
are freely propagating above (below) $2 \mathrm{~mm}$ from the aperture and are not perturbed by streaming plasma coming off the wires (see Fig. 1). This allowed us to more accurately follow the plasma jets using interferometry and estimate their velocity. Note that the apertures are only blocking a small portion of the wire array streaming plasma $(<10 \%)$ and the collimation of the jets is not greatly affected.

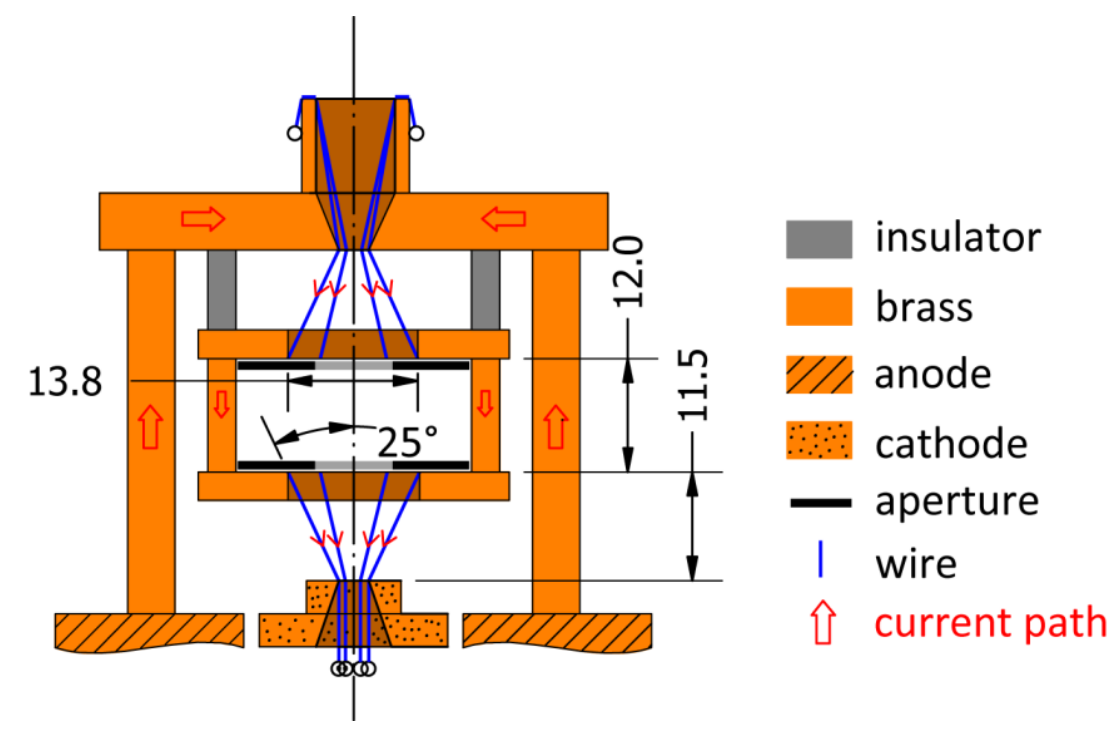

Figure 1. Cross-section of the experimental setup. Two conical wire arrays were used, driven by the same current pulse, to produce counter-propagating plasma flows. The dimensions are in millimeters.

We employed a number of diagnostics to examine the evolution of the jets. The main diagnostic was 4 -frame interferometry with a $5 \mathrm{~ns}$ laser pulse width at $532 \mathrm{~nm}$ and $15 \mathrm{~ns}$ inter-frame separation. The images were recorded with a charge-coupled device (CCD) camera, with a final resolution of the optical system of $\sim 40 \mu \mathrm{m}$. The recorded interferograms were then unfolded using the analysis software IDEA[23] for two-dimensional areal electron density mapping. The four beams were distributed so that they converge at the array center with a separation of $\sim 5^{\circ}$ : each beam has an almost identical viewing axis, allowing us to directly compare the interferograms to one another. A four-frame Extreme Ultraviolet (XUV) gated camera with unfiltered $50 \mu \mathrm{m}$ pinholes was also implemented. Each frame took a $5 \mathrm{~ns}$ exposure, the magnification of the system was $\sim 1$, and the spatial resolution was $\sim 100 \mu \mathrm{m}$. The cut-off energy of the photocathode is $\sim 5$ $\mathrm{eV}$, but diffraction effects become comparable to geometric effects at $\sim 25 \mathrm{eV}$. At lower photon energy, light is diffracted away and hardly reaches the quadrant camera[24].

\section{Experimental results for counter-propagating plasma jet collisions}

The motivation of this work was to create a platform for launching counter-propagating plasma jets using a compact current driver at UCSD to study astrophysics related phenomena. The current driver gave high-quality data despite several limitations of the driver, such as the current amplitude and the driver impedance. The former limits the maximum density achieved in the precursor plasma and the jets, while the latter constrains the inductance and hence the size of the setup. We found that the dimensions shown in Fig. 1 were sufficient, as they gave enough density to be detected by interferometry and have adequate size to use multiple diagnostics simultaneously. As there is abundant work on aluminum wire arrays, we tested our setup using aluminum wires for comparison. Fig. 2 displays a time sequence of unfolded interferograms showing the evolution of the jet interaction. The grey areas in the pictures are products of broken fringes in the interferograms that were not possible to unfold. Each row of 4 frames in Fig. 2 is from the same shot. 
The evolution is as follows: at 200-220 ns the plasma jets stagnate at a position above the center of the experimental setup, indicating that the bottom jet is faster than the top one. The average jet electron density prior to collision can be obtained by dividing the areal density by the jet diameter. For our experiment, we calculated a density of $\sim 3 \times 10^{17} \mathrm{~cm}^{-3}$. The plasma density is well-resolved at the merging region, showing a density jump of $\sim 4$ with respect to the density of the jets. The density jump does not appear to increase considerably throughout the evolution of the interaction. During this time the shock bends, exhibiting a subtle concave curvature. Then, between 236 and 252 ns, it flattens and remains stationary. At approximately $260 \mathrm{~ns}$, the curvature of the shock becomes convex and begins to move down toward the cathode. It is interesting to note that the plasma does not spread sideways along the bisector plane. Instead, the plasma forms a cone-shaped structure (bow shock), along which the bottom plasma jet is diverted. In the second row of Fig. 2, - a different shot- it is seen that the bow shock has fully developed, pointing downwards. This shock moves at a speed of $\sim 20 \mathrm{~km} / \mathrm{s}$, which may be due to differences in the jet speeds and/or densities. The jet speeds were estimated by blocking one jet while allowing the other to freely propagate following a constant density profile (measured by interferometry). In this fashion, we obtained a value of $100-200 \mathrm{~km} / \mathrm{s}$. We confirmed velocity measurements by studying the interaction of a single jet with a solid wall, producing a reverse shock. The speed measured using this method was also found to be in the same range. However, neither method is reliable enough to give a sufficiently accurate measurement: the first is subject to cooling effects, and the second to material ablation from the interacting wall. The only method that could give an accurate value is optical Thomson scattering, which in Ref. [25] has been proven to work in pulsed-power-driven wire array plasmas.

Looking at the first row in Fig. 2, we observe that the opening angle at the base of the bottom jet increases with time. As this is due to thermal expansion at the sound speed, by measuring the radius of the plasma column at different times, we obtain a sound speed of $\mathrm{C}_{\mathrm{s}} \approx 22 \mathrm{~km} / \mathrm{s}$. From this value we can also estimate the plasma temperature using the relation $\mathrm{C}_{\mathrm{s}}{ }^{2}=\gamma(\mathrm{Z}+1) \mathrm{k}_{\mathrm{B}} \mathrm{T} / \mathrm{m}_{\mathrm{i}}$, where $\mathrm{Z}$ is the average ionization state, $\mathrm{m}_{\mathrm{i}}$ is the ion mass and $\gamma$ is the ratio of the specific heats. Then, $\mathrm{T} \sim 20 \mathrm{eV}$ and $\mathrm{Z} \sim 3$, where we have used the approximation $\mathrm{Z} \approx 0.63 \sqrt{ } \mathrm{T}_{\mathrm{eV}}$ [26]. Using our measured value for the sound speed, we find that the Mach number of the jets is $\mathrm{M} \sim 5-10$, which is related to the Euler number by $\mathrm{M}=\mathrm{E}_{\mathrm{u}} \sqrt{ } \gamma$. The other dimensionless parameters prior to collision are calculated as follows. The Reynolds number is $R_{e}=v L / v$, where $v$ is the kinematic ion viscosity (see ref. [27]), and $v$ and $L$ are the characteristic speed and length of the system, respectively. Substituting in the values found above ( $\approx \approx 100 \mathrm{~km} / \mathrm{s}, L \approx 2 \mathrm{~mm}$ ), we find $R_{e}>10^{4}$. The magnetic Reynolds number can be calculated via $R_{e M}=\mu_{0} v L / \eta$, where $\eta$ is the electrical resistivity and can be found from Ref. [27]. We calculate $\mathrm{R}_{\mathrm{e} \mathrm{M}} \approx 20$, meaning that magnetic field advection by the plasma flow is significant and any initial magnetic field produced by the external current could be entrained by the flow. The Peclet number, $\mathrm{P}_{\mathrm{e}}=\mathrm{vL} / \alpha$, where $\alpha$ is the thermal diffusivity (see Ref. [6]), is estimated to be $\mathrm{P}_{\mathrm{e}} \sim 10$. The cooling parameter $(\chi)$ is more complicated to compute, as we have to deal with atomic physics. It is defined as the ratio of radiative cooling time to the hydrodynamic timescale of the jet $\left(\sim \mathrm{R}_{\text {jet }} / \mathrm{C}_{\mathrm{s}}\right)$, i.e. $\chi=\tau_{\text {rad }} / \tau_{\text {hyd }}$. The radiative cooling time $\tau_{\text {rad }}$ can be computed as $\varepsilon_{T} / P_{R}$, where $\varepsilon_{T}$ is the thermal energy density and $P_{R}$ is the radiated power per unit volume. Using the radiative cooling rate tables given in Ref.[28], we estimate the cooling parameter to be $\chi>1$, meaning that the jets are adiabatic. If $\chi<1$, they are said to be radiatively cooled. Using the expression given in Ref. [6] for the localization parameter $\delta$ (the ratio of the particle's mean free path to the jet radius), we find for the ions that $\delta<10^{-2}$, meaning that single jet propagation can be described by hydrodynamics.

The question arises as to whether the actual interaction can also be described by the hydrodynamic equations. One necessary condition is that the localization parameter $\delta$, should be much smaller than 1 . We can estimate the inter-jet ion mean free path from the following equation given in Ref. [21]: 


$$
\approx 5 \times 10^{-13} \frac{A_{Z}^{2}}{Z^{4}} \frac{[\mathrm{v}(\mathrm{cm} / \mathrm{s})]^{4}}{n_{Z}\left(\mathrm{~cm}^{-3}\right)}
$$

Using the aforementioned values of $A_{Z}=27, Z=3$, and $\mathrm{n}_{\mathrm{Z}} \sim 1 \times 10^{17} \mathrm{~cm}^{-3}$, we get an inter-jet ion mean free path on the order of $5-80 \mathrm{~mm}$ for $100-200 \mathrm{~km} / \mathrm{s}$, respectively, meaning that $\delta>1$. Therefore, considering that the plasma is non-magnetized, the interaction region cannot be described by the hydrodynamic equations. Moreover, this means that the plasma jets should not interact at all, and the jets should inter-penetrate each other. It is interesting now to elucidate the mechanisms behind the plasma stagnation at the interaction region. One possibility is that the presence of a magnetic field could cause this effect. If we consider that the shock width is on the order of the Larmor radius, then we should have that $\mathrm{B} \sim 10 \mathrm{~T}$. We have found that $\mathrm{R}_{\mathrm{eM}} \approx 20$, therefore it is plausible that some magnetic field could have been advected by the plasma flows from the array region, where the magnetic field is of the same order of magnitude. Another possibility is that this magnetic field could have been created by (e.g. Weibel) instabilities in the colliding region, but thus far we have not seen any evidence of filamentation that supports this idea. If the shock is supported by magnetic fields, then the ram pressure is on the order of the magnetic pressure $\left(\rho v^{2} \approx B^{2} / 2 \mu_{0}\right)$, giving $B \sim 15 T$ - consistent with the value found above. Therefore, it is possible that the shock is mediated by the magnetic field advected by the plasma flows. In the future we are planning to use miniature magnetic probes to study the advection of magnetic fields.

a) $220 \mathrm{~ns}$

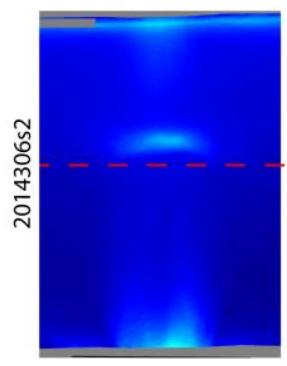

e) $275 \mathrm{~ns}$

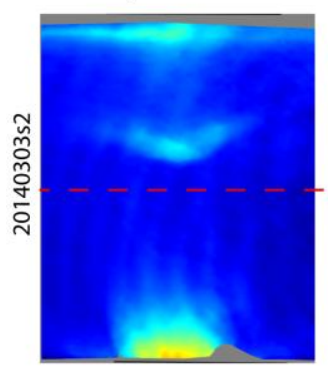

b) $236 \mathrm{~ns}$

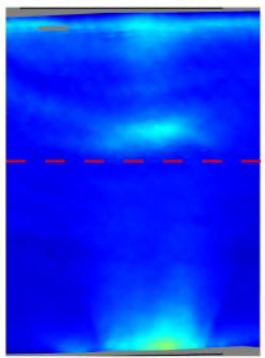

f) $291 \mathrm{~ns}$

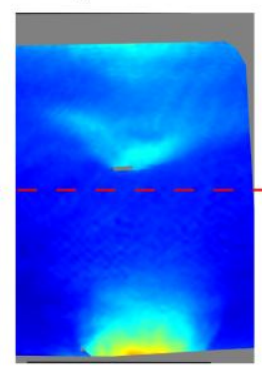

c) $252 \mathrm{~ns}$

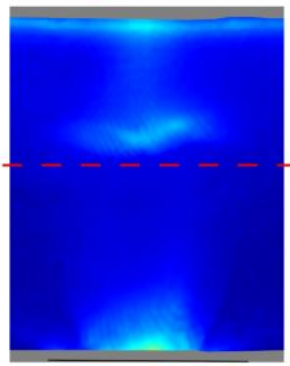

g) $307 \mathrm{~ns}$

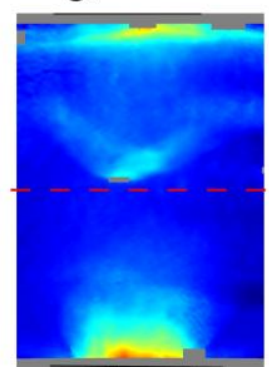

d) $268 \mathrm{~ns}$

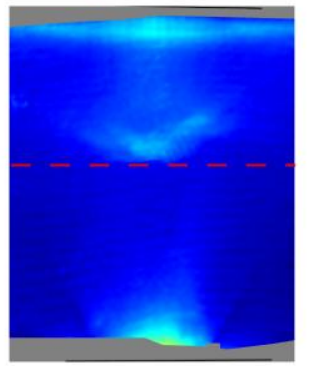

h) $323 \mathrm{~ns}$

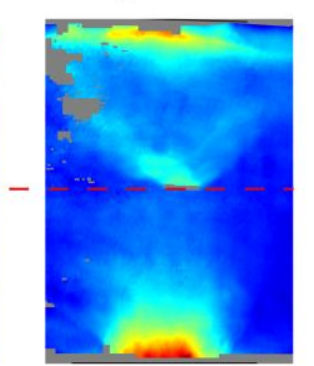

$\mathrm{x} 10^{17} \mathrm{~cm}^{-2}$

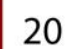

10

5

$<1$

$5 \mathrm{~mm}$

Figure 2. Unfolded interferometry data showing a time sequence of the interaction of aluminum counter-propagating plasma jets. The pictures in each row come from the same shot. The dashed lines are a spatial reference.

XUV imaging of the colliding plasma jets is displayed in Fig. 3. The camera's viewing axis was $45^{\circ}$ with respect to the laser diagnostic line of sight. Similar structures can be seen in the XUV images and the interferometry data (Fig. 2), demonstrating that the shock wave's structure is axially symmetric. This was also corroborated by rotating the array $90^{\circ}$ and using interferometry. From Fig. 3, at $227 \mathrm{~ns}$ it is seen that 
the top jet is more collimated than the bottom one, which can be seen subtly in the interferometry data between 236-268 ns (Fig. 2). This could explain the formation of the bow shock and why it moves downwards. However, the collimation process is still uncertain. Later in time, the cone-shape structure is seen to develop as observed with interferometry. Comparing Fig. 3 at 312 ns with the interferometry data (Fig. 2, 307-323 ns) we can see that there is only a slight increase in density at the tip of the shock compared to the wings. Conversely, in the XUV images, emission coming from the apex is more significant. This implies that the plasma is being shocked at that point and that the bottom jet is being diverted by this shock, forming the conical shape.

a) $227 \mathrm{~ns}$

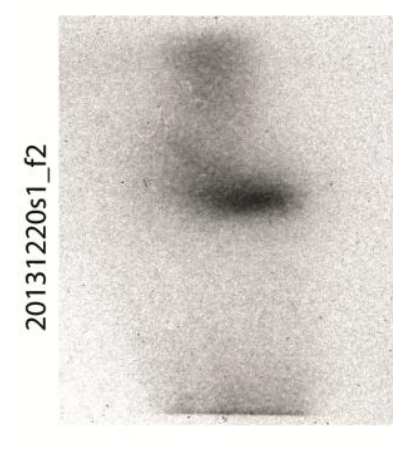

b) $312 \mathrm{~ns}$

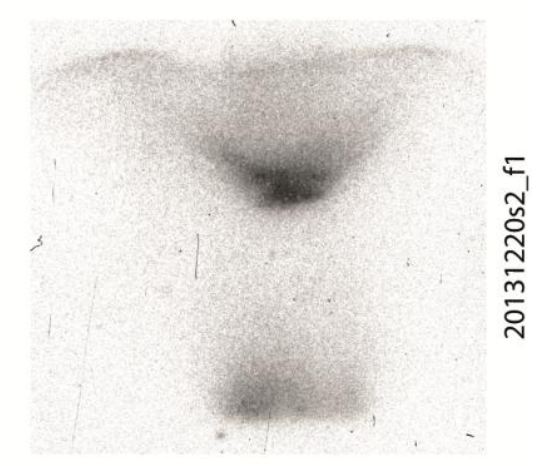

$5 \mathrm{~mm}$

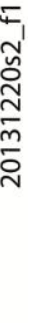

Figure 3. Gated XUV pinhole images of the collision of two aluminum jets.

\section{Astrophysical relevance}

It is theorized that radiative cooling effects in astrophysics are responsible for different sorts of instabilities and morphologies in jets and shocks. In order to access this more radiative regime, we used the same experimental set-up shown in Fig. 1 but with a higher-Z wire material, $\mathrm{Cu}$. As the atomic number of copper (29) is larger than aluminum's (13), cooling due to radiation is expected to be stronger than the Al-Al case. This was first tested using $\mathrm{Cu}$ wires in both arrays. As can be seen from the results presented in Fig. 4, a very thin shock is formed compared to $\mathrm{Al}$, but, when the bow shock starts to develop at about the same time as Al ( 250 ns) it quickly fragments and becomes unstable, preventing further instability grow. After $250 \mathrm{~ns}$, interferometry only shows a diffuse plasma; those results are not shown here. Then, in order to study the case when only the more-collimated jet is radiatively cooled, we changed just the top array to Cu. Some experimental results are displayed in Fig. 5. From these images we can see that a bow shock also develops, moving downward at a speed of $\sim 20 \mathrm{~km} / \mathrm{s}$ as in the Al-Al case, but as the jet radiatively cools, the jet pressure decreases and the bow shock becomes more collimated. Also note that the contact discontinuity between the two materials is much sharper than in case of Al-Al jets, and as result it becomes unstable and fragments. At this point in time, we also observe that the bow shock tip narrows and a head-like structure develops. The cooling parameter for a copper jet can also be estimated by using the radiative cooling rates from ref. [28]; we find that $\chi \sim 0.02$. This indicates that radiative cooling is dynamically important and could explain the differences seen in Fig. 4 and 5 compared to the Al-Al case (Fig. 2). 


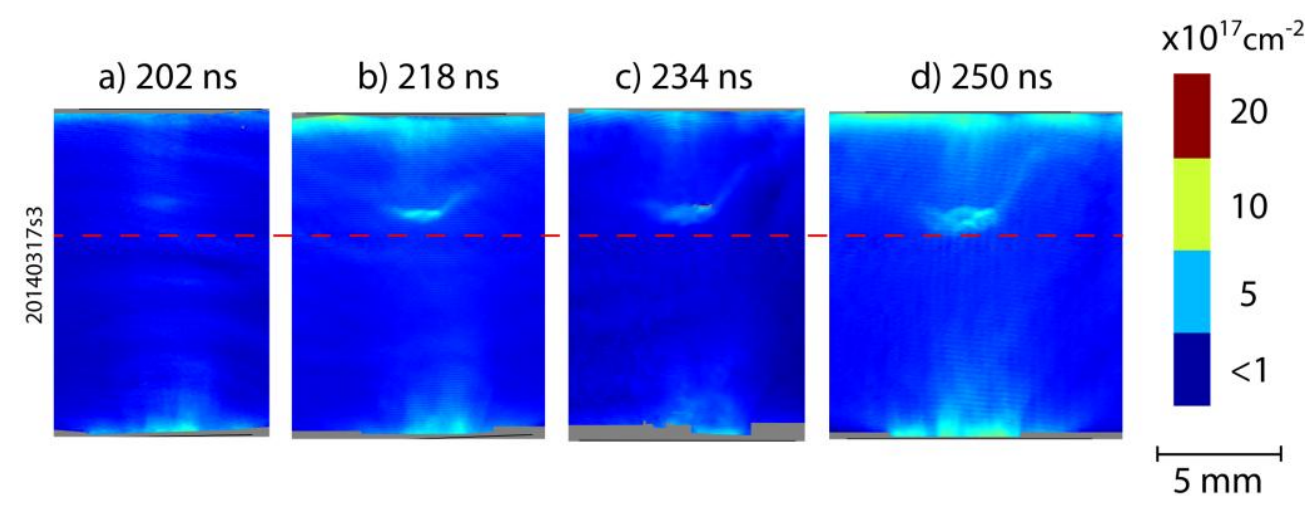

Figure 4. Areal electron density maps showing a time sequence of the interaction of copper counter-propagating plasma jets.

Qualitative similarities can be seen between these data and the Herbig-Haro objects (for example HH 34 [3]), including the bow shock, clumpy features and leading nose cone. Several authors have studied the narrowing of the jet tips using numerical methods[4,5], finding that besides radiative cooling, a toroidal magnetic field could have a significant effect on narrowing the jet heads due to pinch forces. The signature of a magnetic field embedded in the jets could give critical information regarding the formation of YSO jets, a process that is not yet well understood. The region where the jets form is usually inaccessible as its scale is below observational limits, and also because it is usually obscured by other star formation processes. As a result, analytical models and numerical simulations that describe the origin and collimation of the jets have not been compared with observational data. The only information available is on the jet itself and its interaction with the interstellar medium. Therefore, any evidence regarding the formation process of the jets would be extremely useful, potentially helping to distinguish between hydrodynamic and MHD jets.

In order to hold relevance to astrophysics, the similarity criteria must be established. Table 1 summarizes the dimensionless parameters found in this work compared to the ones found in YSO jets [29]. Some parameters such as $\mathrm{Pe}$ and $\mathrm{Re}_{\mathrm{M}}$ are not as large as the ones found in YSO jets; this means that the hydrodynamics and MHD equations will not be accurate describing the plasma behavior at very small scales and thus similarity between the systems will not be reliable at those regions. Nevertheless, the equations describing the system and similarity will be valid at larger scales (see Ref. [6] for more details). Notice that we have not included a full analysis of the radiation losses comparing an astrophysical system with our laboratory system. We leave this analysis to future work. Nevertheless, it is important to point out the simplicity of studying its effect by using materials with higher atomic numbers.

Table 1. Laboratory-astrophysical scalability comparison.

\begin{tabular}{|c|c|c|}
\hline Dimensionless parameter & Experiment & Young stellar objects \\
\hline $\mathrm{M}$ (Mach number) & $5-10$ & $5-40$ \\
\hline $\mathrm{R}_{\mathrm{e}}$ (Reynolds number) & $>10^{4}$ & $>10^{8}$ \\
\hline $\mathrm{R}_{\mathrm{eM}}$ (Magnetic Reynolds number) & $\sim 20$ & $>10^{15}$ \\
\hline $\mathrm{P}_{\mathrm{e}}$ (Peclet number) & $\sim 10$ & $>10^{7}$ \\
\hline$\delta$ (localization parameter) & $\sim 10^{-2}$ & $<10^{-6}$ \\
\hline$\chi$ (cooling parameter) & $0.02-10$ & $0.01-100$ \\
\hline
\end{tabular}


This work may be considered as a basis where the influence of the relevant parameters on jet collimation and propagation can be studied experimentally and compared with astrophysical systems, provided adequate scalability. In the future we will perform a thorough study on what triggers the narrowing of the bow shock tip by changing the density contrast and the radiative cooling rate, and compare the experimental data with numerical simulations. These results could, for the first time, shed light on the interaction between astrophysical plasma jets and a background medium, providing a benchmark for astrophysical modelling and simulation.

a) $291 \mathrm{~ns}$

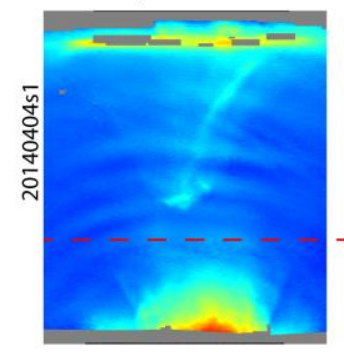

b) $307 \mathrm{~ns}$

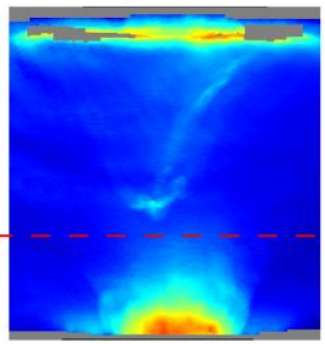

e)

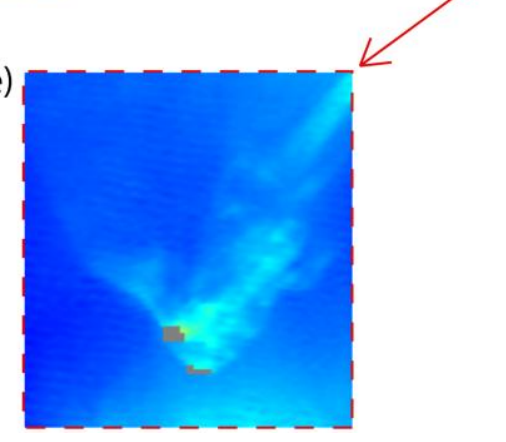

c) $323 \mathrm{~ns}$

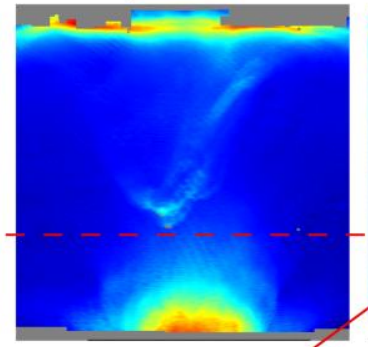

d) $339 \mathrm{~ns}$

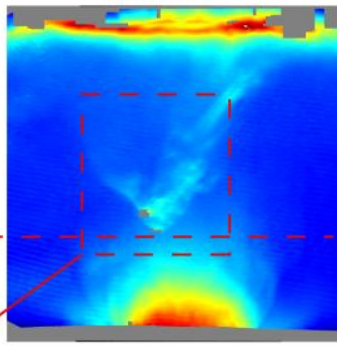

$\mathrm{x} 10^{17} \mathrm{~cm}^{-2}$

20

10

5

$<1$

Figure 5. a)-d) Unfolded interferometry data showing a time sequence of the interaction of two counter-propagating plasma jets. The top jet was made of copper and the bottom one of aluminum. e) A close up of the leading bow shock at $339 \mathrm{~ns}$.

\section{Summary and future plan}

We have shown that it is possible to study interesting physics relevant to astrophysics on a compact current driver producing only $200 \mathrm{kA}$, compared to the $1 \mathrm{MA}$ generators generally used for these studies. The use of a smaller driver is often an advantage as it is easier to operate and the shot rate is usually higher.

We produced two supersonic, counter-propagating plasma flows that move at $\sim 200 \mathrm{~km} / \mathrm{s}$ (Mach number 10) towards each other and collide. During their interaction, a bow shock is produced that propagates at 20 $\mathrm{km} / \mathrm{s}$ towards the cathode of the array. We believe the bow shock forms as one of the jets is less collimated than the other, which acts as a background medium. Aluminum wires were tested first; it was observed that the bow shock is adiabatic and that it does not seem to break up for a considerable period of time (>50 ns). On the contrary, when one of the arrays was made of copper and the other of aluminum, significant differences are seen. As radiative cooling in copper is more significant, the bow shock was observed to collapse into a thin shell and then fragment, forming clumpy features. Simultaneously, the head (tip) of the bow shock is seen to get narrow as the shock moves at a similar speed observed in the Al-Al case. This effect qualitatively resembles $\mathrm{HH}$ objects. We are planning to carefully study this bow shock behavior and its development in the future by changing the critical parameters such as radiative cooling, density contrast, and magnetic field. This work should be used to benchmark astrophysical models and numerical codes. 
The dimensionless numbers on single jet propagation were found to be adequate for the use of hydrodynamics or ideal MHD. However as $\delta>1$, to use these equations the jet interaction itself will require further analysis, thus only a significant magnetic field where the Larmor radius is smaller than the shock structure length would guarantee the applicability of the MHD equations. As the inter-jet ion mean free path is larger than the characteristic scale length of the jet, the shock is not collision-mediated. We discussed the possibility of a magnetic field-mediated shock, either advected by the plasma flow $\left(\mathrm{R}_{\mathrm{eM}} \sim 20\right)$ or produced by instabilities. It was found that in order to support the ram pressure, the magnetic field should be $\sim 15 \mathrm{~T}$, which is on the order of magnitude of the magnetic field found at the wire array position. To validate this we are planning to perform magnetic field measurements using miniature magnetic probes, which will give us an estimation of the magnetic field amplitude as well as its time dependence.

We also leave as future work further analysis of the inter-jet ion collisionality. More precise measurement of jet speed and temperature will help us accurately estimate the ion mean free path. This topic could be especially useful for developing a small-scale experiment capable of examining collisionless shock physics.

\section{Acknowledgements}

The authors would like to thank Prof. Sergei Krasheninnikov for useful discussions and comments. This work was partially funded by Department of Energy Grant No. DE-SC0001063.

\section{References}

[1] N. Soker, M. Livio, Disks and jets in planetary nebulae, Astrophys. J. 421 (1994) 219-224.

[2] M. Begelman, R. Blandford, M. Rees, Theory of extragalactic radio sources, Rev. Mod. Phys. 56 (1984) 255-351.

[3] B. Reipurth, J. Bally, Herbig-Haro flows: Probes of early stellar evolution, Annu. Rev. Astron. Astrophys. 39 (2001) 403-455.

[4] J. Blondin, B. Fryxell, A. Konigl, The structure and evolution of radiatively cooling jets, Astrophys. J. 360 (1990) 370.

[5] A. Frank, D. Ryu, Effects of cooling on the propagation of magnetized jets, Astrophys. J. 494 (1998) L79-L83.

[6] D. Ryutov, R. Drake, J. Kane, Similarity criteria for the laboratory simulation of supernova hydrodynamics, Astrophys. J. 518 (1999) 821-832.

[7] D. Ryutov, R.P. Drake, B.A. Remington, Criteria for scaled laboratory simulations of astrophysical MHD phenomena, Astrophys. J. Supl. Ser. 127 (2000) 465-468.

[8] P. Hartigan, The visibility of the Mach disk and the bow shock of a stellar jet, Astrophys. J. 339 (1989) 987.

[9] P. Hartigan, J. Raymond, L. Hartmann, Radiative bow shock models of Herbog-Haro objects, Astrophys. J. 316 (1987) 323-348. 
[10] D.D. Ryutov, B. a. Remington, H.F. Robey, R.P. Drake, Magnetohydrodynamic scaling: From astrophysics to the laboratory, Phys. Plasmas. 8 (2001) 1804. doi:10.1063/1.1344562.

[11] B. a. Remington, J. Kane, R.P. Drake, S.G. Glendinning, K. Estabrook, R. London, et al., Supernova hydrodynamics experiments on the Nova laser, Phys. Plasmas. 4 (1997) 1994. doi:10.1063/1.872341.

[12] H. Takabe, H. Nagatomo, Recent studies of laser produced plasmas, Plasma Phys. Control. Fusion. 41 (1999) A75-A79.

[13] H.F. Robey, J.O. Kane, B. a. Remington, R.P. Drake, O. a. Hurricane, H. Louis, et al., An experimental testbed for the study of hydrodynamic issues in supernovae, Phys. Plasmas. 8 (2001) 2446. doi:10.1063/1.1352594.

[14] a. Ciardi, S. V. Lebedev, J.P. Chittenden, D.J. Ampleford, S.N. Bland, B.S. Bott, et al., Modeling Magnetic Tower Jets in the Laboratory, Astrophys. Space Sci. 298 (2005) 277-286. doi:10.1007/s10509-005-3947-8.

[15] S. Lebedev, J. Chittenden, Laboratory astrophysics and collimated stellar outflows: The production of radiatively cooled hypersonic plasma jets, Astrophys. J. 564 (2002) 113-119.

[16] S. V Lebedev, a Ciardi, D.J. Ampleford, S.N. Bland, S.C. Bott, J.P. Chittenden, et al., Production of radiatively cooled hypersonic plasma jets and links to astrophysical jets, Plasma Phys. Control. Fusion. 47 (2005) B465-B479. doi:10.1088/0741-3335/47/12B/S33.

[17] S. V. Lebedev, D. Ampleford, A. Ciardi, S.N. Bland, J.P. Chittenden, M.G. Haines, et al., Jet Deflection via Crosswinds: Laboratory Astrophysical Studies, Astrophys. J. 616 (2004) 988-997. doi:10.1086/423730.

[18] D. Ampleford, S. Lebedev, A. Ciardi, Formation of working surfaces in radiatively cooled laboratory jets, Astrophys. Space Sci. (2005) 241-246.

[19] F. Suzuki-Vidal, S. V. Lebedev, M. Bocchi, M. Krishnan, G. Swadling, G. Burdiak, et al., Experimental study of shock waves from the interaction of a supersonic plasma jet with an ambient gas, Shock Waves. 22 (2012) 249-251. doi:10.1007/s00193-012-0360-4.

[20] N.L. Kugland, D.D. Ryutov, P.-Y. Chang, R.P. Drake, G. Fiksel, D.H. Froula, et al., Self-organized electromagnetic field structures in laser-produced counter-streaming plasmas, Nat. Phys. 8 (2012) 809-812. doi:10.1038/nphys2434.

[21] H.-S. Park, D.D. Ryutov, J.S. Ross, N.L. Kugland, S.H. Glenzer, C. Plechaty, et al., Studying astrophysical collisionless shocks with counterstreaming plasmas from high power lasers, High Energy Density Phys. 8 (2012) 38-45. doi:10.1016/j.hedp.2011.11.001.

[22] S.C. Bott, D.M. Haas, R.E. Madden, U. Ueda, Y. Eshaq, G. Collins, et al., 250 kA compact linear transformer driver for wire array z-pinch loads, Phys. Rev. Spec. Top. - Accel. Beams. 14 (2011) 050401.

[23] M. Hipp, J. Woisetschläger, P. Reiterer, T. Neger, Digital evaluation of interferograms, Measurement. 36 (2004) 53-66. doi:10.1016/j.measurement.2004.04.003. 
[24] S.N. Bland, D.J. Ampleford, S.C. Bott, S. V. Lebedev, J.B. a. Palmer, S. a. Pikuz, et al., Extreme ultraviolet imaging of wire array z-pinch experiments, Rev. Sci. Instrum. 75 (2004) 3941. doi:10.1063/1.1787926.

[25] a. J. Harvey-Thompson, S. V. Lebedev, S. Patankar, S.N. Bland, G. Burdiak, J.P. Chittenden, et al., Optical Thomson Scattering Measurements of Plasma Parameters in the Ablation Stage of Wire Array Z Pinches, Phys. Rev. Lett. 108 (2012) 145002. doi:10.1103/PhysRevLett.108.145002.

[26] R.P. Drake, High-Energy-Density Physics: Fundamentals, Inertial Fusion, and Experimental Astrophysics, 2006.

[27] M.G. Haines, A review of the dense Z -pinch, Plasma Phys. Control. Fusion. 53 (2011) 93001. doi:10.1088/0741-3335/53/9/093001.

[28] D. Post, R. Jensen, C. Tarter, Steady-state radiative cooling rates for low-density, high-temperature plasmas, At. Data Nucl. Data Tables. 20 (1977) 397.

[29] A. Ciardi, laboratory studies of astrophysical plasmas, Lect Notes Phys. 793 (2010) 31-50. doi:10.1007/978-3-642-02289-0. 